\title{
Second harmonic nanoparticles in imaging applications
}

\author{
Ye Pu ${ }^{* a}$, Chia-Lung Hsieh ${ }^{\mathrm{a}, \mathrm{b}}$, Rachel Grange ${ }^{\mathrm{a}, \mathrm{c}}$, Xin Yang ${ }^{\mathrm{a}}$, Ioannis Papadopoulos ${ }^{\mathrm{a}}, \mathrm{Jae}-\mathrm{Woo} \mathrm{Choi}^{\mathrm{a}}$, \\ and Demetri Psaltis ${ }^{\mathrm{a}}$ \\ ${ }^{a}$ Optics Laboratory, School of Engineering, EPFL STI LO, BM 4.107, Station 17, \\ 1015 Lausanne, Switzerland \\ ${ }^{\mathrm{b}}$ Department of Electrical Engineering, California Institute of Technology, 1200 East California \\ Boulevard, MC 136-93, Pasadena, California 91125, USA \\ ${ }^{\mathrm{c}}$ Multiphoton Laboratory, Institute of Applied Physics, Friedrich Schiller University, Albert- \\ Einstein-Str. 15, 07745 Jena, Germany \\ ye.pu@epfl.ch
}

\begin{abstract}
Nanocrystals with second harmonic response is a new class of nonlinear optical nanoprobes with dramatically different properties from fluorescent agents. Compared with two-photon fluorescence, second harmonic generation is an ultrafast, lossless, and coherent process. In particular, the absence of photobleaching and emission intermittency in the optical response of the second harmonic nanoparticles is likely to complement the fluorescent agents widely used today in many imaging applications. Furthermore, the coherent emission from the second harmonic generation process provides unique opportunities for the application of coherence domain techniques that are not available with fluorescent agents. We review the application of the second harmonic nanocrystals in imaging applications, especially those pertaining to biomedicine.
\end{abstract}

Keywords: nonlinear optics, second harmonic generation, nanocrystals, multiphoton imaging, two-photon microscopy, three-dimensional, molecular tagging, two-photon fluorescence

\section{INTRODUCTION}

One of the most important tasks and the greatest challenges in biomedical imaging is to achieve a contrast between the molecules of interest and the crowd of irrelevant ones, or the "background". Fluorescent agents such as organic dyes, green fluorescent protein (GFP), and quantum dots (QDs) have been the standard approach for this purpose because of their excellent brightness and wide availability [1]. They are increasingly imaged through two-photon excitation in recent years as ultrafast laser technologies mature, owing to the superior penetration depth of near infrared light. However, a number of issues associated with fluorescence, particularly photobleaching and emission intermittency, complicate the observations $[2,3]$.

An optical analog of two-photon fluorescence (TPF) in terms of contrast forming is second harmonic generation (SHG), a nonlinear frequency-doubling process. SHG is strictly specific to materials with noncentrosymmetric structures and vanishes in all other types of materials. A sharp contrast is formed in the second harmonic image between materials of noncentrosymmetric structures and other species when they are excited at a fundamental frequency. Therefore, SHG satisfies the requirement to serve as a mechanism of contrast for biomedical imaging applications. Recently, endogenous SHG has been employed to image certain biological tissue structures with excellent clarity [4-6]. However, endogenous SHG does not provide molecular-specific information, a vital component in modern biological research. Nanoprobes with SHG response and affinity to specific biomolecules are therefore intriguing in the search for new imaging modalities.

We developed a new type of contrast agent employing second harmonic generation (SHG) rather than two-photon fluorescence (TPF). Figure 1 (a) illustrates the principle of SHG nanoprobes. Essentially, a SHG nanoprobe consists of a non-centrosymmetric nanocrystal which, under an intense optical excitation, gives rise to second harmonic due to a nonlinear polarization. Since the physical dimension of the nanocrystal is much smaller than the wavelength involved, The phase-matching condition is universally satisfied, and thus the harmonic radiation is in all direction.

Active Photonic Materials IV, edited by Ganapathi S. Subramania, Stavroula Foteinopoulou, Proc. of SPIE Vol. 8095, 80950E - (C) 2011 SPIE · CCC code: 0277-786X/11/\$18 · doi: 10.1117/12.894567 
As shown in Fig. 1 (b), SHG is a completely different physical process from TPF. The process of SHG only involves a virtual electron energy transition that lasts less than a femtosecond during the simultaneous annihilation of two excitation photons and emission of one photon with exactly twice the input photon energy. Therefore, SHG is a lossless, coherent, ultrafast process. TPF, on the other hand, involves real electron energy transition through the simultaneous absorption of two excitation photons, which lasts a few nanoseconds before the energy is released in the emission of a photon with less than twice the input photon energy. The TPF process is thus lossy, with energy lost in vibrational relaxation. Virtually all fluorescent agents are subject to gradual lost of emission intensity (photobleaching) and emission intermittency ("blinking"). SHG nanoprobes thus possess many properties that are complementary to that of TPF probes, among which the most notable ones are the absence of photobleaching and signal blinking.
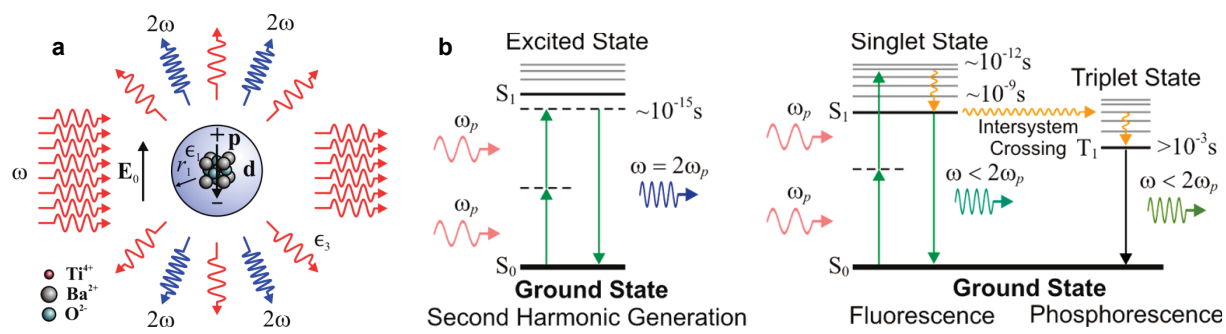

Figure 1. (a) Principle of SHG nanoprobes. A nanocrystal of noncentrosymmetric structure is excited by an external optical field. The nonlinear polarization gives rise to second harmonic radiation. Since the physical dimension of the nanocrystal is much smaller than the wavelengths involved in the process, the second harmonic emission is in all direction due to a universal phase-matching. (b) Comparison between SHG and TPF in electron transition diagram. In SHG, electrons undergo only an ultrafast, lossless, virtual energy transition, annihilate two photons and generate one photon with twice the frequency simultaneously. The TPF process, on the other hand, involves real electron energy transitions, is lossy, and subject to photobleaching and emission intermittency.

\section{MATERIALS AND PROPERTIES OF SECOND HARMONIC NANOPROBES}

A key issue in the development of SHG nanoprobes is the choice of suitable nonlinear optical material. A number of nonlinear optical nanocrystals that are capable of SHG have been investigated in previous studies [7-11]. Our work focuses on tetragonal $\mathrm{BaTiO}_{3}$ nanocrystals [12-15]. $\mathrm{BaTiO}_{3}$ is a well-known material widely used in both photonics and electronics. As the solution-phase synthesis of ferroelectric nanocrystals matures [16], tetragonal $\mathrm{BaTiO}_{3}$ nanocrystals have been commercially available. Pertaining to imaging applications, we refer to these SHG nanoparticles as second harmonic radiation imaging probes (SHRIMPs). Some of the unique optical properties of SHRIMPs are demonstrated in the following experiments.

Emission stability is crucial in the long-term observation of dynamic events. The stability of SHG emission in a sample of $100-\mathrm{nm} \mathrm{BaTiO}_{3}$ nanocrystals was measured using a two-photon laser scanning microscope. A continuous scanning was performed over a period of five hours, and the SHG intensity of a single SHRIMP as a function of time is plotted in Fig. 2 (a). The signal intensity remains constant in the entire period without any fluctuation routinely found in fluorescence measurements. Similar results based on $\mathrm{KTiOPO}_{4}$ nanocrystals were also reported in an earlier work [10].
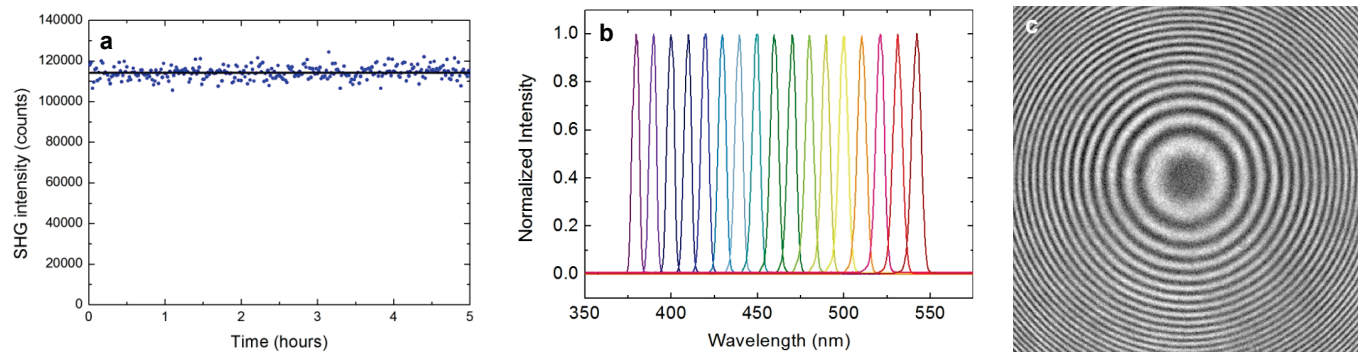

Figure 2. (a) Emission stability of SHG showing the lack of photobleaching and emission blinking. The blue dots are measurements and the black line is a linear fit. (b) Normalized emission spectra of a SHG nanocrystal when the excitation was tuned from 720 to $980 \mathrm{~nm}$, indicating excellent flexibility in the choice of excitation frequency. (c) Interference pattern between a planar and a spherical second harmonic wave independently generated from a BBO and a quartz crystal, respectively, revealing the coherent nature of SHG processes. 
Because of the non-resonant nature of the SHG process, the second harmonic radiation in SHRIMPs is well tunable while the excitation frequency changes [9]. This was demonstrated by measuring the emission spectrum while the excitation laser was tuned from 720 to $980 \mathrm{~nm}$, as shown in Fig. 2 (b). The SHG output wavelength follows exactly half that of the excitation, with a narrow bandwidth of $5 \mathrm{~nm}$. As a comparison, the spectrum of two-photon fluorescence, often more than $50 \mathrm{~nm}$ in bandwidth, does not change with the excitation frequency. The narrow, tunable SHG spectrum not only provides flexibility in the choice of excitation wavelength but also allows for efficient suppression of background emissions such as autofluorescence.

Although the photon annihilation and emission in optically separated crystals are independent processes, the second harmonic radiations are coherent and interfere with each other regardless the underlying materials that generate the harmonics. Figure 2 (c) shows an interference pattern between a second harmonic spherical wave, generated in a quartz plate, and a second harmonic plane wave, generated in a beta barium borate (BBO) crystal. This coherence property enables the detection of SHG nanoparticles using interferometric techniques, based on which we have demonstrated a new holographic principle [12].

Lithium niobate $\left(\mathrm{LiNbO}_{3}\right)$ is another highly versatile photonic material with an unusually high SHG coefficient. In addition to $\mathrm{BaTiO}_{3}$ nanoparticles, we also investigated the synthesis of $\mathrm{LiNbO}_{3}$ nanowires [17]. Figure 3 (a) shows the scanning electron microscope (SEM) image of a single isolated $\mathrm{LiNbO}_{3}$ nanowire. The nanowire was synthesized using $\mathrm{Nb}_{2} \mathrm{O}_{5}$ and $\mathrm{LiOH}$ via a hydrothermal route at a temperature of $150^{\circ} \mathrm{C}$. X-ray and electron diffraction pattern (data hot shown) suggested that the nanowires have a trigonal crystal structure. The SHG measurement corresponding to this wire is plotted in Fig. 3 (b), which matches theoretical prediction well. The strongly polarization-dependent SHG response was applied in the modulation of second harmonic emissions by dielectrophoretic (DEP) forces in a microfluidic channel.
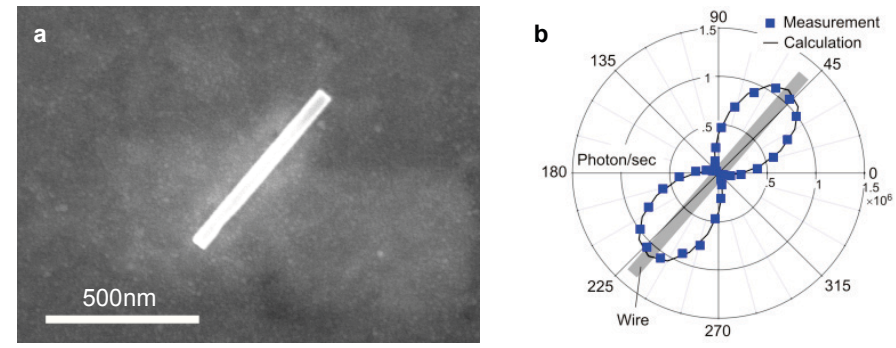

Figure 3. (a) SEM of a single $\mathrm{LiNbO}_{3}$ nanowire deposited on ITO coated glass. The nanowire is $50 \mathrm{~nm}$ wide and $700 \mathrm{~nm}$ long. (b) The polarization response of the SHG from a single LiNbO3 nanowire. The blue square dots are measurement data, and the black curve is calculation results.

\section{MOLECULAR TARGETING OF SECOND HARMONIC NANOPROBES}

In order to enable molecularly specific imaging for biological applications using the $\mathrm{BaTiO}_{3}$ SHRIMPs, we further developed protocols to conjugate them to antibodies [14] in a four-step process (Fig. 4). First, amine groups was attached on the surface of the $\mathrm{BaTiO}_{3}$ nanocrystal using 3-Aminopropyltriethoxysilane (APTES), providing a highly versatile anchoring point for further attachments of other molecules on the surface. Second, the surface amine group was activated using Sulfosuccinimidyl-4-(N-maleimidomethyl)cyclohexane-1-carboxylate (Sulfo-SMCC). Third, Immunoglobulin G (IgG) molecules was reduced using Dithiothreitol (DTT) to obtain sulfhydryl-tethered antibody. Finally, the activated $\mathrm{BaTiO}_{3}$ particles bind to the sulfhydryl group on the IgG molecules. 


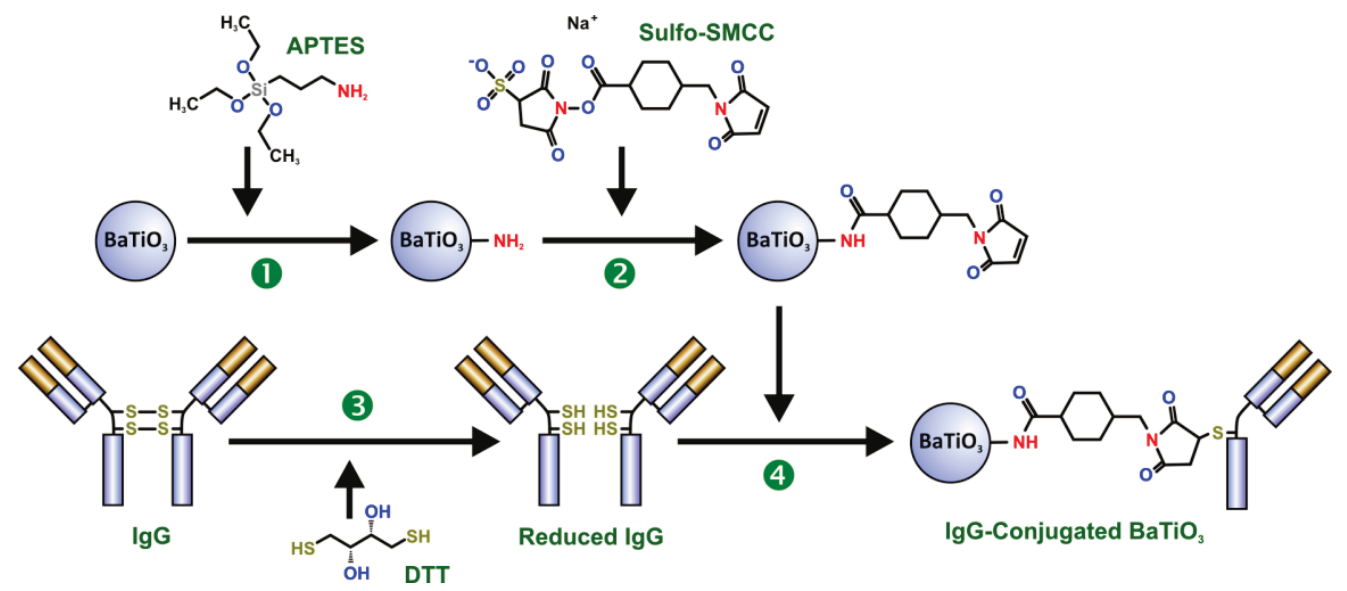

Figure 4. Principle of $\mathrm{BaTiO}_{3}$-antibody conjugation. Four main steps are involved. Step 1: functionalization of the $\mathrm{BaTiO}_{3}$ nanocrystal surface with amine functional group using aminosilane (APTES); Step 2: activation of the surface amine group using Sulfo-SMCC; Step 3: antibody (IgG) reduction using DTT; Step 4: binding of antibody to the activated $\mathrm{BaTiO}_{3}$ nanocrystals.

The scheme of conjugation was demonstrated in an indirect staining process: the target molecule was first labeled with a primary antibody, which is then recognized by a secondary-antibody conjugated to SHRIMPs. Tests in a protein microarray show that the bioconjugation scheme has a high staining specificity. As shown in Fig. 5 (a), the SHRIMPs conjugated with donkey anti-mouse IgG specifically bind to mouse IgG with very low unspecific binding. In a cell imaging setup, the efficiency of the conjugation was demonstrated in Fig 5 (b) and (c) using human leukocyte antigen (HLA) class I surface antigen as the target molecule. The conjugated SHRIMPs clearly bound to the membrane of the HeLa cells stained with primary antibody as seen in Fig. 5 (b), while the control in Fig. 5 (c) shows virtually no binding because the cells were not stained with the primary antibody. In addition, our cytotoxicity tests shown in Fig. 5 (d) suggest that the $\mathrm{BaTiO}_{3}$ nanoparticles are highly biocompatible for the use as an imaging probe. Cells remain viable and show no sign of death at a particle concentration up to $1 \times 10^{10}$ particles $/ \mathrm{mL}$.
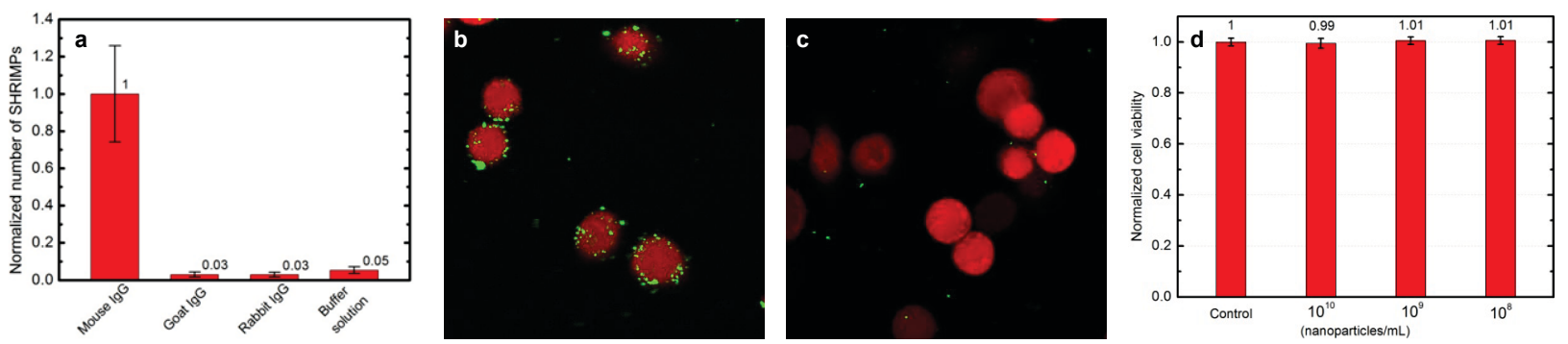

Figure 5. (a) Binding specificity of antibody-conjugated $\mathrm{BaTiO}_{3}$ nanoparticles measured with a protein microarray. (b) Confocal section images of HeLa cells labeled with the antibody-conjugated $\mathrm{BaTiO}_{3}$ nanoparticles. The cells were first labeled with mouse anti-human IgG specific to the HLA class I molecules present on the cell membrane, followed by the SHRIMPs conjugated with an anti-mouse IgG. (c) A control sample where the cells were not labeled with primary antibody under otherwise identical conditions; The SHG images are shown in the green channel, and the TPF images are shown in the red channel. (d) Cytotoxicity test results of $\mathrm{BaTiO}_{3}$ nanoparticles, suggesting that $\mathrm{BaTiO}_{3}$ nanoprobes are highly biocompatible.

\section{ENHANCEMENT OF SHG IN PLASMONIC NANOCAVITIES}

To further increase the detection sensitivity of the SHG nanoprobes, we also developed plasmonic core-shell nanocavities based on nonlinear optical $\mathrm{BaTiO}_{3}$ nanocrystals as the core material [15]. At resonance, the plasmonic shell around the $\mathrm{BaTiO}_{3}$ core greatly concentrates the excitation field locally, creating a significantly enhanced second harmonic response in the nonlinear optical core media. The synthesis protocol as illustrated in Fig. 6 was based on seeded condensation of gold on the surface of $\mathrm{BaTiO}_{3}$ and followed earlier procedures for $\mathrm{SiO}_{2} / \mathrm{Au}$ core-shells [18]. Briefly, the surface of $\mathrm{BaTiO}_{3}$ nanoparticles was first functionalized with amine group using APTES. Small gold 
nanoparticles sized 2-3 nm was then adsorbed onto the amine-coated surface. Finally, the gold-seeded $\mathrm{BaTiO}_{3}$ nanocrystals was injected into a Chloroauric acid where seeded condensation of gold is initiated using a proper reducer.

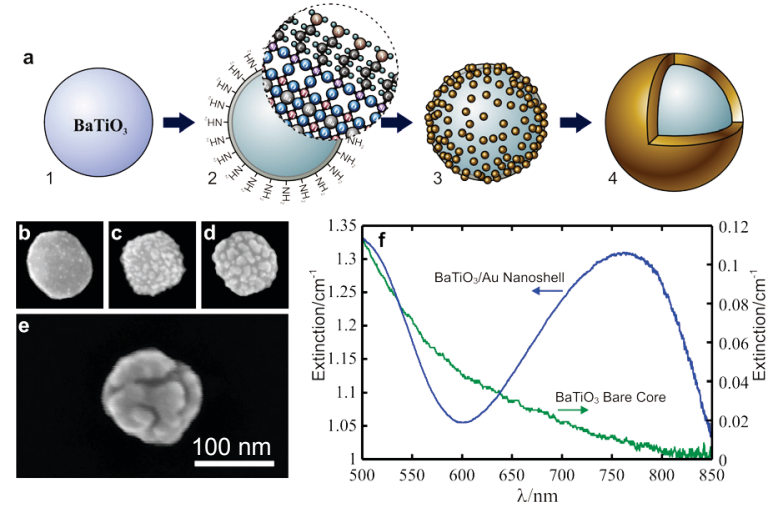

Figure 6. (a) Principle of the synthesis of $\mathrm{BaTiO}_{3} / \mathrm{Au}$ core-shell nanocavities. Step1, bare BaTiO3 core; Step 2, surface functionalization using aminosilane (APTES), a simplified atomic arrangement on the core surface is shown in the dashed circle; Step 3, adsorption of 2-3 nm gold nanoparticles on the surface of the core; Step 4, formation of the complete gold shell through seeded condensation. (b) - (d) SEM image of three intermediate synthesis steps. (e) SEM image of a typical synthesis product. (f) Extinction spectrum of the core-shell nanocavities (blue) and that of the bare cores (green). The resonance peak is clearly identified in the core-shell nanocavities.

Figure 7 shows the experimental results of the SHG enhancement measurements, where (a) and (b) is the baseline SHG scattering cross-section of bare $\mathrm{BaTiO}_{3}$ particles, (c) and (d) is the cross-section of the nonlinear optical plasmonic nanocavities. In all measurements, signal particles were identified by SEM and shown in the inset, and $\gamma$ indicates the polarization angle of the excitation beam. The measurements indicate that the second harmonic radiation power is enhanced roughly 540 times. The results are in good agreement with the theoretical prediction, which also suggested potentials to reach more than 3000 times when the right geometry is well formed.
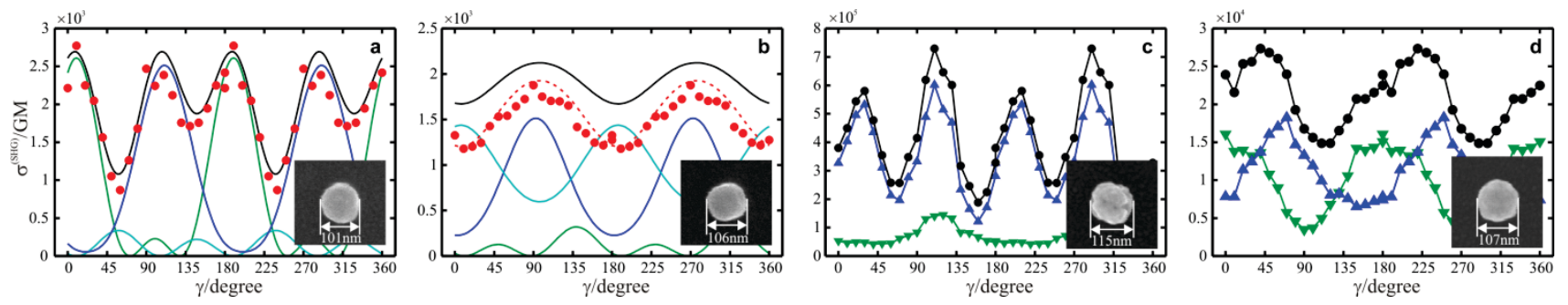

Figure 7. (a) and (b) Calculated and measured SHG scattering cross-sections corresponding to the SEM image of the bare $\mathrm{BaTiO}_{3}$ nanocrystal shown in the respective insets. Red dots, measurements. Blue, green, and cyan solid curves, calculated x-, y-, and z-components of the cross-section. Black solid curve, total cross-section. Red dotted curve, calculations treating $\mathrm{z}$ and $\mathrm{x}-\mathrm{y}$ components equally. (c) and (d) Measurements of the SHG scattering cross-section corresponding to the $\mathrm{BaTiO}_{3} / \mathrm{Au}$ core-shell nanostructure shown in the respective insets. Blue up triangles, $\mathrm{x}-$ polarization. Green down triangles, y-polarization. Black circles, total cross-section. According to calculations, measurements in (c) is on-resonance and measurements in (d) is off-resonance. The factor of on-resonance enhancement is 540 .

\section{APPLICATIONS IN BIOMEDICAL IMAGING}

The coherent emission in the SHG scattering process provides a unique opportunity for the application of interferometric imaging techniques for dynamic imaging in biological systems with fine resolution in all three dimensions and time. As a first step to achieve this goal, we have demonstrated a new holographic principle, the harmonic holography [9], which uses independently generated second harmonics from the SHG nanocrystals and a dedicated bulk SHG crystal as the signal and reference waves, respectively. As illustrated in Fig. 8, this principle was later applied in the three-dimensional imaging of small $\mathrm{BaTiO}_{3}$ nanoparticle clusters as SHRIMPs in HeLa cells [10]. The $\mathrm{H}^{2}$ microscope is essentially a special type of Mach-Zehnder interferometer that involves independently generated second harmonics in each arm. The reference arm contains a beta Barium Borate (BBO) crystal and a telescope expander to generate a SHG reference. The 
signal arm contains an infinity-conjugated microscope to collect the second harmonics produced by the nanocrystals embedded in the sample. An electron multiplying charge-coupled device (EMCCD) attached with a SHG band-pass filter captures the interference between the signal and the reference at the second harmonic frequency.

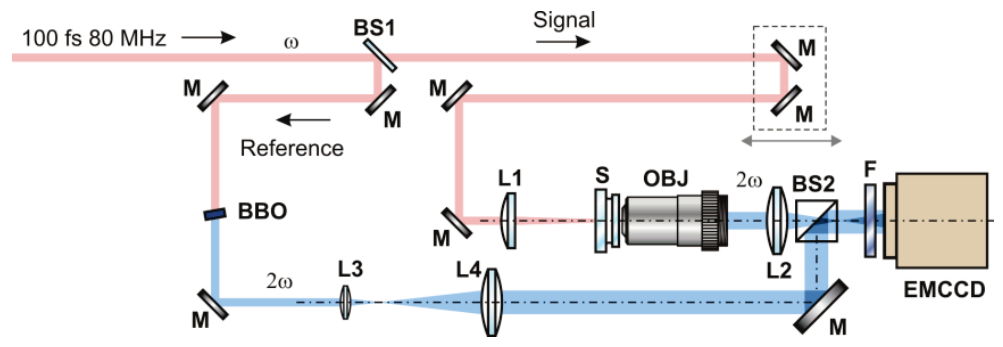

Figure 8. Experimental setup for $\mathrm{H}^{2}$ microscopy. BS1 splits the laser into signal and reference beams. In the signal arm, the sample S containing SHRIMPs is excited by the signal beam lightly focused by L1, and the second harmonic radiation is collected by the microscope composed of OBJ and L2 in an infinity-conjugated configuration. The EMCCD is placed away from the imaging plane. A band-pass filter is placed in front of the EMCCD to remove the excitation from the SHG signal. In the reference arm, the beam is frequency-doubled by a BBO crystal and expanded by a telescope constructed with L3 and L4. A variable delay line (dashed rectangle) is used to ensure the temporal overlapping of the reference and signal waves. The signal and reference beams are combined collinearly by BS2 and therefore an on-axis digital hologram is recorded on the EMCCD. BS1 and BS2, beam splitters; M, mirror; L1 - L4, lenses; S, sample; OBJ, microscope objective; F, SHG band-pass filter.

Figure 9 (a) shows the superposition of the bright field transmission image of a cell (red channel) and the SHG image from SHRIMPs (green channel) taken by a two-photon laser-scanning microscope. Six SHRIMP clusters were identified and labeled with numbers, although only SHRIMPs that are on-focus in the particular image slice are visible. Figure 9 (b) - (d) shows selected image planes reconstructed from a single hologram of the same area at distinct axial positions, where on-focus SHRIMP images are marked with arrows. Figure 9 (e) shows the normalized axial intensity profiles of the six clusters, demonstrating the three-dimensionality of the reconstructed images and the good signal-to-noise ratio (SNR). Molecularly specific three-dimensional imaging can be achieved with this approach once properly conjugated SHG particles are used to stain the cells.
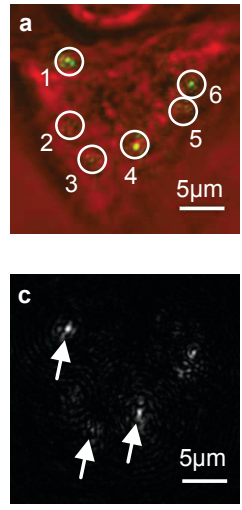
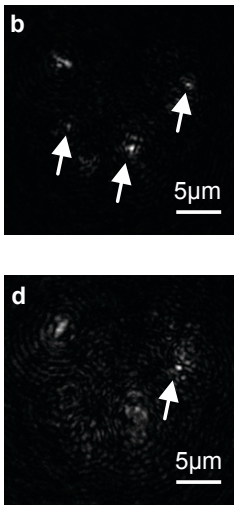

e

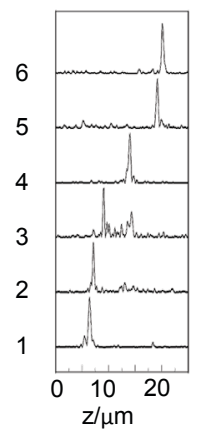

Figure 9. (a) Superposition of the bright field transmission image of a HeLa cell (red channel) and the SHG image of SHRIMPs (green channel) taken by a conventional microscope. Six SHRIMP clusters were identified, some of which were out of focus and cannot be seen in one slice. (b) - (d) Image planes reconstructed from a single $\mathrm{H}^{2}$ frame at relative depths of $0,3.1$, and $6.2 \mu \mathrm{m}$ respectively. The white arrows show the SHRIMPs that are on focus. The SHRIMP labeled with number 4 is a big cluster so that it is bright both in (b) and (c). (e) Normalized intensity line profiles of the six clusters.

Light scattering and possible endogenous SHG present challenges in the imaging of SHRIMPs in tissues, especially in the collagen-rich region. We have performed laser-scanning microscopy of $\mathrm{BaTiO}_{3}$ nanoparticles in the tail of a live mouse [20] where endogenous SHG is known to be strong. In the experiments, an anesthetized mouse was injected 5-10 $\mu \mathrm{L}$ water solution of $300 \mathrm{~nm}$ nanoparticles around the middle of the tail. The mouse was then placed under a two-photon laser-scanning microscope with the tail immersed in water, as shown in Fig. 10 (a). Despite the significant endogenous 
SHG form the dermal collagen, we was able to identify the second harmonic signals from the nanoparticles as deep as $100 \mu \mathrm{m}$ below the surface of the tail with a SNR of $\sim 10$ [Fig. 10 (b)].

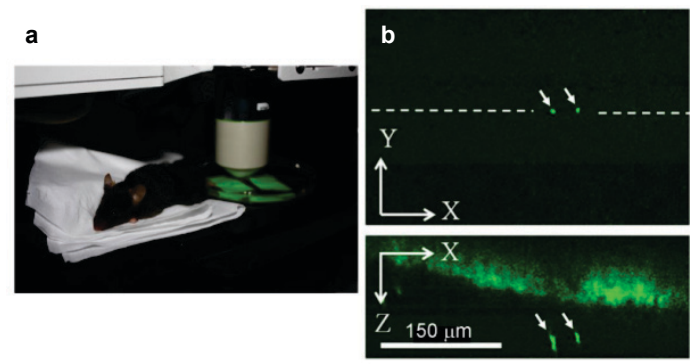

Figure 10. Imaging of SHG nanoparticles in live tissue. (a) Sample diagram (b) Slices of image planes in a threedimensional image volume of the SHRIMPs embedded $100 \mu \mathrm{m}$ inside a mouse tail tissue obtained with a two-photon laser-scanning microscope. Top panel: x-y cut; Bottom: x-z cut. Endogenous SHG can be seen in the x-z plane. The arrows indicate the SHRIMPs in both views.

In tissue imaging, the resolution and image SNR greatly suffers deep inside the tissue due to the strong scattering. Focusing light through turbid media is therefore a highly sought after capability for imaging applications in deep tissues. However, its implementation remains challenging. It has been shown that digital holographic phase-conjugation can achieve efficient focusing through turbid media [19], though the problem of creating a coherent beacon inside the highly scattering medium remains unresolved. Phase-conjugation based on $\mathrm{H}^{2}$ provides a unique solution to this problem owing to its coherent contrast that can be potentially molecular-specific. We have demonstrated efficient focusing of light through a turbid medium using $\mathrm{H}^{2}$ phase-conjugation [21]. Figure 11 depicts the principle of $\mathrm{H}^{2}$ digital phase conjugation, where a variant of the $\mathrm{H}^{2}$ microscopy shown in Fig. 8 is used with a single SHRIMP in the sample as a light beacon. The system first record the phase of the SHG light field emitted from the SHRIMP and scattered by a turbid layer [Fig. 11 (a)]. Then, a light field with the conjugated phase is created by the SLM and sent back through the turbid layer along the same path. The scattering of the turbid layer is canceled by the conjugated phase, and a focus is reconstructed at the location of the SHRIMP.
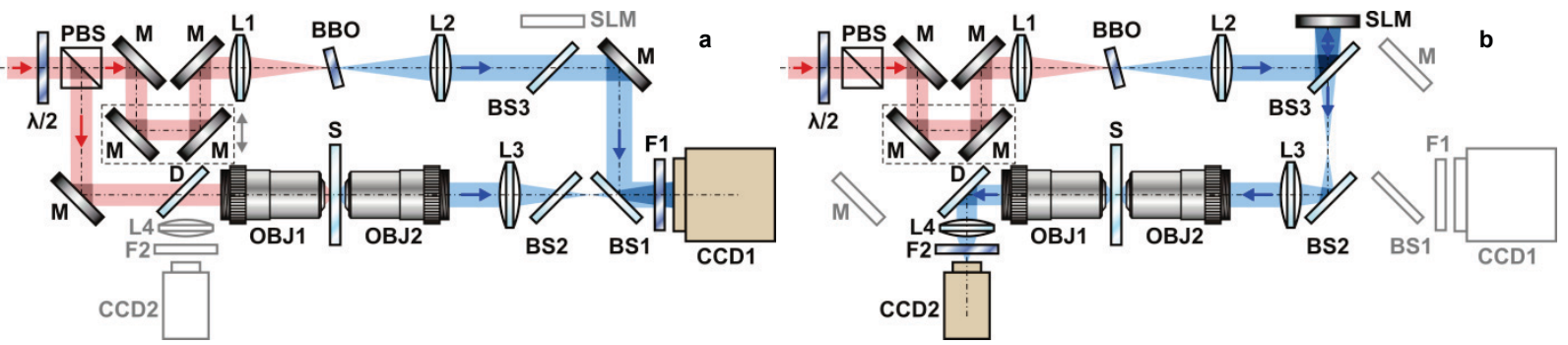

Figure 11. Experimental setup for digital phase conjugation with SHG nanoparticles. (a) $\mathrm{H}^{2}$ recording of the scattered light field. The sample S contains isolated SHG nanoparticles behind (with respect to CCD1) a turbid layer. The digital camera CCD1 records the holograms formed by the scattered SHG light field and the second harmonic reference generated independently by a BBO. (b) Digital phase conjugation. The second harmonic reference is directed to the SLM where the conjugated wave front is recreated, goes through the same turbid layer, and focuses onto CCD2. $\lambda / 2$, half wave plate; PBS, polarization beam splitter; M, mirror; D, dichroic mirror; L1 - L4, lens; OBJ1 and OBJ2, microscope objectives; S, sample; BS1 - BS3, beam splitters; F1 and F2, SHG band-pass filter. In both panels, components in light gray is unused.

Figure 12 shows the experimental results of the $\mathrm{H}^{2}$ phase conjugation. The conjugated phase is shown in Fig. 12 (a) where a severely distorted wave front is implied by the random phase map. When this phase pattern was projected on the SLM, the light focused at the position of the SHRIMP as shown in Fig. 12 (b). As a control experiment, a phase map captured without the turbid layer was projected on the SLM, in which case no focus was identified. Plotted in Fig. 12 (d) is the intensity profile of the conjugated focus, which has a full width half maximum (FWHM) of $2.30 \mu \mathrm{m}$, very close to the diffraction limit of the system $(1.95 \mu \mathrm{m})$. 

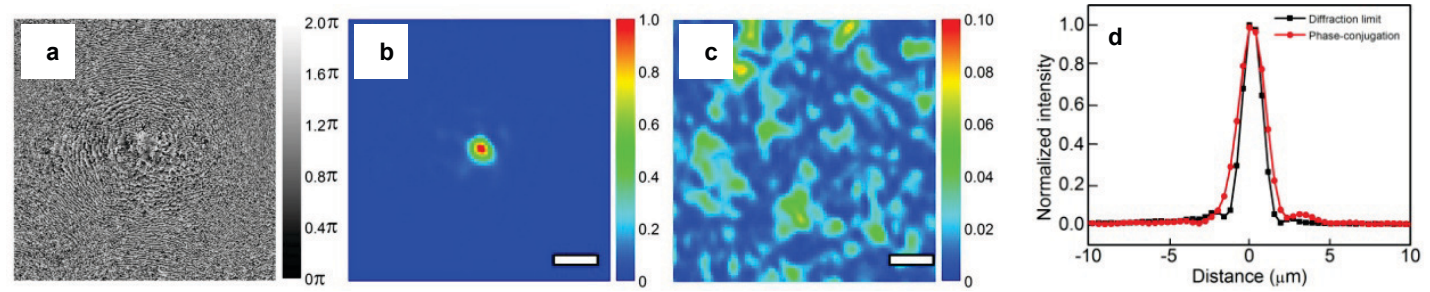

Figure 12. Digital phase conjugation through a turbid medium. (a) Conjugated phase pattern of the scattered SHG field. (b) Normalized intensity image (captured by CCD2) of the phase-conjugated focus through the turbid medium by using the phase pattern shown in (a). (c) Normalized intensity image of the highly distorted focus without phase-conjugation. (d) Comparison of the phase-conjugated focus with the diffraction limit. Red: normalized intensity profile of the phaseconjugated focus. Black: normalized intensity profile of the diffraction limited focus. The FWHMs of the phaseconjugated and diffraction limit are $2.30 \mu \mathrm{m}$ and $1.95 \mu \mathrm{m}$ respectively.

In order to apply the $\mathrm{H}^{2}$ phase conjugation technique to imaging, a means of scanning the conjugated focus is required. We demonstrated in a later work [22] that the reestablished focus can be scanned in the vicinity of the original position due to the optical memory effect of disordered media [23]. The experimental setup was the same as that is illustrated in Fig. 11, albeit with CCD2 replaced by a photomultiplier (PMT). The target was a photolithographic pattern of gold on a glass plate, as seen in Fig. 13 (a). A SHRIMP beacon was placed in the center of the pattern for $\mathrm{H}^{2}$ recording, and a ground glass diffuser was used as a turbid layer. Scanning the phase-conjugated focus over the target and collecting the light transmitted through with the PMT recreated the target pattern [Fig. 13 (b)]. In the control experiment without phase-conjugation, no pattern was reconstructed [Fig. 13 (c)]. Figure 13 (d) plots an intensity profile along the dashed line in Fig. 13 (b), indicating good SNR and contrast. With more sensitive SHG nanoprobes, such as those demonstrated in [15], it is tangible to achieve both recording and phase-conjugation from the same side, which would enable uninvasive tissue imaging in an unprecedented depth.
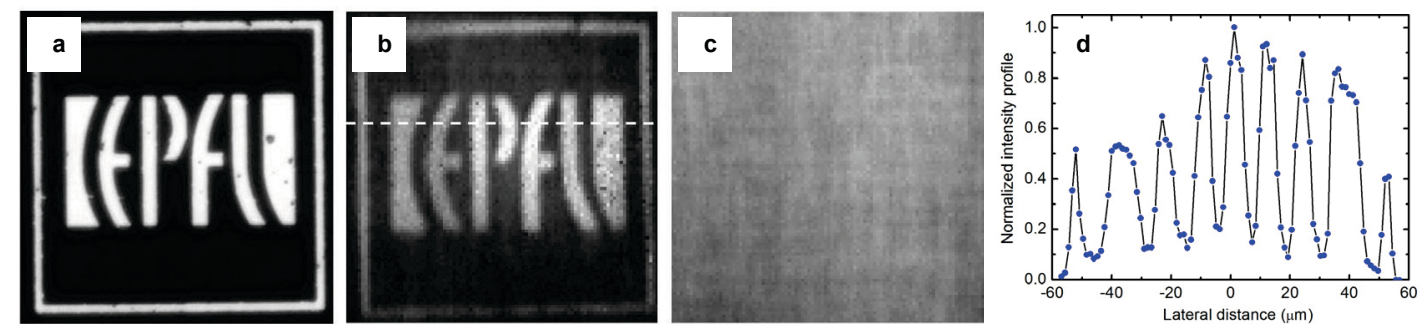

Figure 13. (a) Wide-field transmission image of the target, a 130-nm thick gold pattern on a glass substrate prepared by photolithography. The bright region indicates the transparent area. (b) Phase-conjugate scanning image of the target. The target pattern is clearly resolved. (c) Scanning image of the same target without phase conjugation. The image is completely scrambled. (d) Intensity profile along the dashed line in (b) showing a good contrast.

\section{CONCLUSION}

Imaging probes to achieve efficient, molecular-specific contrast play a vital role in the field of biomedical imaging. We have demonstrated that nanocrystals capable of second harmonic generation, or SHRIMPs, serve as imaging probe that are dramatically different form fluorescent agents widely used today. The absence of photobleaching and emission intermittency makes the SHG imaging probes an excellent complement to fluorescent probes. We also developed protocols for the surface functionalization and bioconjugation of the SHG nanoparticles, which paved the way toward a wide range of biomedical imaging applications. We further demonstrated that the sensitivity of the SHG nanoprobes can be significantly enhanced by enclosing them in a resonant plasmonic nanocavity. Moreover, the coherence property of SHG enables three-dimensional imaging with molecular-specific contrast. We demonstrated such potentials through harmonic holography imaging in cells. We also show that digital phase conjugation through harmonic holography holds great promise in the imaging through turbid media, a highly sought-after technique mandated by deep-tissue imaging. 


\section{REFERENCES}

[1] B. N. G. Giepmans, S. R. Adams, M. H. Ellisman, and R. Y. Tsien, “The fluorescent toolbox for assessing protein location and function”, Science 312, 217-224 (2006).

[2] R. M. Dickson, A. B. Cubitt, R. Y. Tsien, and W. E. Moerner, "On/off blinking and switching behaviour of single molecules of green fluorescent protein", Nature 388, 355-358 (1997).

[3] G. H. Patterson, and D. W. Piston, "Photobleaching in two-photon excitation microscopy", Biophys. J. 78, 2159-2162 (2000).

[4] A. Zoumi, A. Yeh, B. J. Tromberg, "Imaging cells and extracellular matrix in vivo by using second-harmonic generation and two-photon excited fluorescence", Proc. Natl. Acad. Sci. USA 99, 11014-11019 (2002).

[5] W. R. Zipfel, R. M. Williams, R. Christie, A. Y. Nikitin, B. T. Hyman, W. W. Webb, "Live tissue intrinsic emission microscopy using multiphoton-excited native fluorescence and second harmonic generation”, Proc. Natl. Acad. Sci. USA 100, 7075-7080 (2003).

[6] P. J. Campagnola, L. M. Loew, "Second-harmonic imaging microscopy for visualizing biomolecular arrays in cells, tissues and organisms", Nat. Biotechnol. 21, 1356-1360 (2003).

[7] J. C. Johnson, H. Q. Yan, R. D. Schaller, P. B. Petersen, P. D. Yang, and R. J. Saykally, "Near-field imaging of nonlinear optical mixing in single zinc oxide nanowires", Nano Lett. 2, 279-283 (2002).

[8] L. Bonacina, Y. Mugnier, F. Courvoisier, R. Le Dantec, J. Extermann, Y. Lambert, V. Boutou, C. Galez, and J. P. Wolf, "Polar Fe( $\left(\mathrm{IO}_{3}\right)_{3}$ nanocrystals as local probes for nonlinear microscopy", Appl. Phys. B 87, 399-403 (2007).

[9] Y. Nakayama, P. J. Pauzauskie, A. Radenovic, R. M. Onorato, R. J. Saykally, J. Liphardt, and P. D. Yang, "Tunable nanowire nonlinear optical probe", Nature 447, 1098-1101 (2007).

[10] L. Le Xuan, C. Zhou, A. Slablab, D. Chauvat, C. Tard, S. Perruchas, T. Gacoin, P. Villeval, J. F. Roch, "Photostable second-harmonic generation from a single $\mathrm{KTiOPO}_{4}$ nanocrystal for nonlinear microscopy", Small 4, 1332-1336 (2008).

[11] A. V. Kachynski, A. N. Kuzmin, M. Nyk, I. Roy, and P. N. Prasad, "Zinc oxide nanocrystals for nonresonant nonlinear optical microscopy in biology and medicine", J. Phys. Chem. C 112, 10721-10724 (2008).

[12] Y. Pu, M. Centurion, and D. Psaltis, "Harmonic holography: a new holographic principle". Appl. Opt. 47, A103-A110 (2008).

[13] C. L. Hsieh, R. Grange, Y. Pu, and D. Psaltis, "Three-dimensional harmonic holographic microcopy using nanoparticles as probes for cell imaging", Opt. Express 17, 2880-2891 (2009).

[14] C. L. Hsieh, R. Grange, Y. Pu, and D. Psaltis, "Bioconjugation of barium titanate nanocrystals with immunoglobulin G antibody for second harmonic radiation imaging probes", Biomat. 31, 2272-2277 (2010).

[15] Y. Pu, R. Grange, C. L. Hsieh, and D. Psaltis, "Nonlinear optical properties of core-shell nanocavities for enhanced second-harmonic generation", Phys. Rev. Lett. 104, 207402 (2010).

[16] S. O'Brien, L. Brus, and C. B. Murray, "Synthesis of monodisperse nanoparticles of barium titanate: Toward a generalized strategy of oxide nanoparticle synthesis", J. Am. Chem. Soc. 123, 12085-12086 (2001).

[17] R. Grange, J. W. Choi, C. L. Hsieh, Y. Pu, A. Magrez, R. Smajda, L. Forro, and D. Psaltis, "Lithium niobate nanowires synthesis, optical properties, and manipulation", Appl. Phys. Lett. 95, 143105 (2009).

[18] S. J. Oldenburg, R. D. Averitt, S. L. Westcott, N. J. Halas, "Nanoengineering of optical resonances", Chem. Phys. Lett. 288, 243-247 (1998).

[19] M. Cui and C. H. Yang, "Implementation of a digital optical phase conjugation system and its application to study the robustness of turbidity suppression by phase conjugation", Opt. Express 18, 3444-3455 (2010).

[20] R. Grange, T. Lanvin, C. L. Hsieh, Y. Pu, and D. Psaltis, "Imaging with second-harmonic radiation probes in living tissue", Biomed. Opt. Express 2, 2532-2539 (2011).

[21] C. L. Hsieh, Y. Pu, R. Grange, and D. Psaltis, "Digital phase conjugation of second harmonic radiation emitted by nanoparticles in turbid media", Opt. Express 18, 12283-12290 (2010).

[22] C. L. Hsieh, Y. Pu, R. Grange, G. Laporte, and D. Psaltis, "Imaging through turbid layers by scanning the phase conjugated second harmonic radiation from a nanoparticle", Opt. Express 18, 20723-20731 (2010).

[23] S. Feng, C. Kane, P. Lee, and A. D. Stone, "Correlations and fluctuations of coherent wave transmission through disordered media", Phys. Rev. Lett. 61, 834-837 (1988). 